\title{
Surface Characterization of Porous Nanomaterials in Environmental Applications by Scanning Electron Microscopy
}

\author{
Go-Woon Lee ${ }^{1,3}$, Ji Hee Kwon ${ }^{1}$, Sung-Chan Jang ${ }^{2.3}$, Changhyun Roh², Kwangshik Myung ${ }^{1}$, and Yun \\ Suk Huh ${ }^{3, *}$ \\ 1. R\&D Platform Center, Korea Institute of Energy Research (KIER), Daejeon, Korea. \\ 2. Advanced Radiation Technology Institute (ARTI), Korea Atomic Energy Research Institute (KAERI), \\ Jeonbuk, Korea. \\ 3. Department of Biological Engineering, Biohybrid Systems Research Center (BSRC), Inha University, \\ Incheon, Korea.
}

Considering the excellent properties of three dimensional (3D) porous graphene framework and Prussian blue (PB), a combination of macro porous graphene network with PB might give enhanced removal capacity of radioactive cesium (137Cs)[1]. Although there have been many reports of PB decorated organic/inorganic composites for Cs removal applications, synthesis of the hetero structured 3D porous $\mathrm{PB} /$ graphene composite is scarce in the literature[2].

In previous work, we present a simple and general method to fabricate $\mathrm{PB}$ /reduced graphene oxide foam (RGOF) via one-step hydrothermal method for the efficient removal of 137Cs. This macro porous network allowed rapid cesium ion diffusion into three dimensionally connected PB, while the interconnected graphene sheets provided excellent mechanical integrity and served a high adsorption capacity $(18.67 \mathrm{mg} / \mathrm{g})$ to the PB. An adsorption isotherm was fitted well by the Langmuir model with a linear regression correlation value of 0.97 . The 3D macro porous adsorbents showed excellent removal efficiency of over 99.5\% 137Cs from contaminated water (87.49 Bq/g)[3].

To characterize the Ca-alginate beads, The SEM images were acquired using an FE-SEM (NNS450, FEI) at various acceleration voltage of 5 to $15 \mathrm{kV}$. The FTIR spectra were recorded using a Jasco FT/IR6600. The XRD patterns were collected using a Bruker D2 PHASER (Germany) diffractometer with Cu $\mathrm{K} \alpha$ radiation. The BET surface area and average pore diameter were obtained from the N2 adsorption/desorption isotherm using a fully automatic physisorption analyzer (ASAP 2020, Tristar). The XPS measurements were obtained using a Thermo Scientific, K-Alpha electron spectrometer with an Al X-ray source. UV-vis analysis were carried out using a V770 (JASCO) spectrophotometer. The mechanical properties were measured using micro-indenter measurements (Noise Is a Signal (NIS), customized). The inductively coupled plasma mass spectrometry (ICP-MS) measurements were carried out using a PerkinElmer ELAN6100. The radioactive cesium activity was measured using a High Purity Germanium (HPGe) detector (Canberra, USA).

Especially the surface analysis of this porous nanomaterials by scanning electron microscopy meet with a difficulty to get a clear cross-sectional SE image. The porous nanomaterial, Ca-alginate beads and other composite such as alginate/AIP clay composite, P-MSC composite were hard to get a clear crosssection of sample. The material was soft texture as marshmallow which cause the structure collapse by blade cutting. Other problem was electron beam damage on the sample. The electron microscopy beam source occurs shrinkage and carbon contamination of the samples. 
In this study, we present successful example of surface characterization of porous nanomaterials in environmental applications by field-emission scanning electron microscopy. Technical description of sample treatment has been demonstrated.

\section{References:}

[1] Parajuli, D. et al, Comparative study of the factors associated with the application of metal hexacyanoferrates for environmental Cs decontamination. Chem. Eng. J. 283(2016), p. 1322-1328. [2] Mihara, Y. et al, Adsorption kinetic model of alginate gel beads synthesized micro particle-prussian blue to remove cesium ions from water. J. Water Process Eng. 10(2016), 9-19.

[3] Sung-Chan Jang et al, Porous three-dimensional graphene foam/Prussian blue composite for efficient removal of radioactive 137Cs. Scienttificreports, 5(2015), 17510.

[4] Sung-Chan Jang et al, Synergistically strengthened 3D micro-scavenger cage adsorbent for selective removal of radioactive cesium. Scienttificreports, 6(2016), 38384.

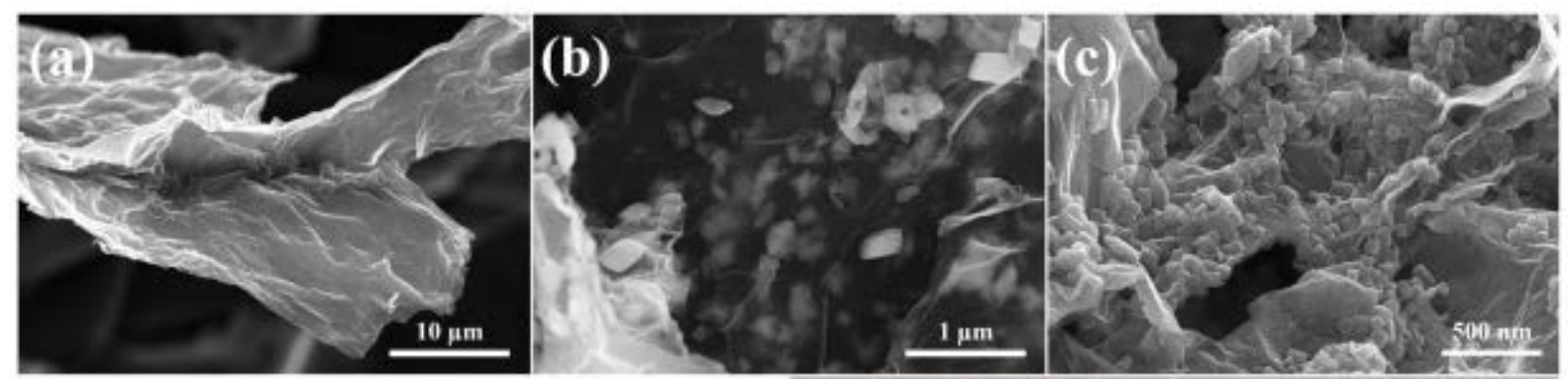

Figure 1. SEM images of the (a) GO, (b) $\alpha-\mathrm{FeOOH} / \mathrm{GO}$, and (c) PB/RGOF composite[3].
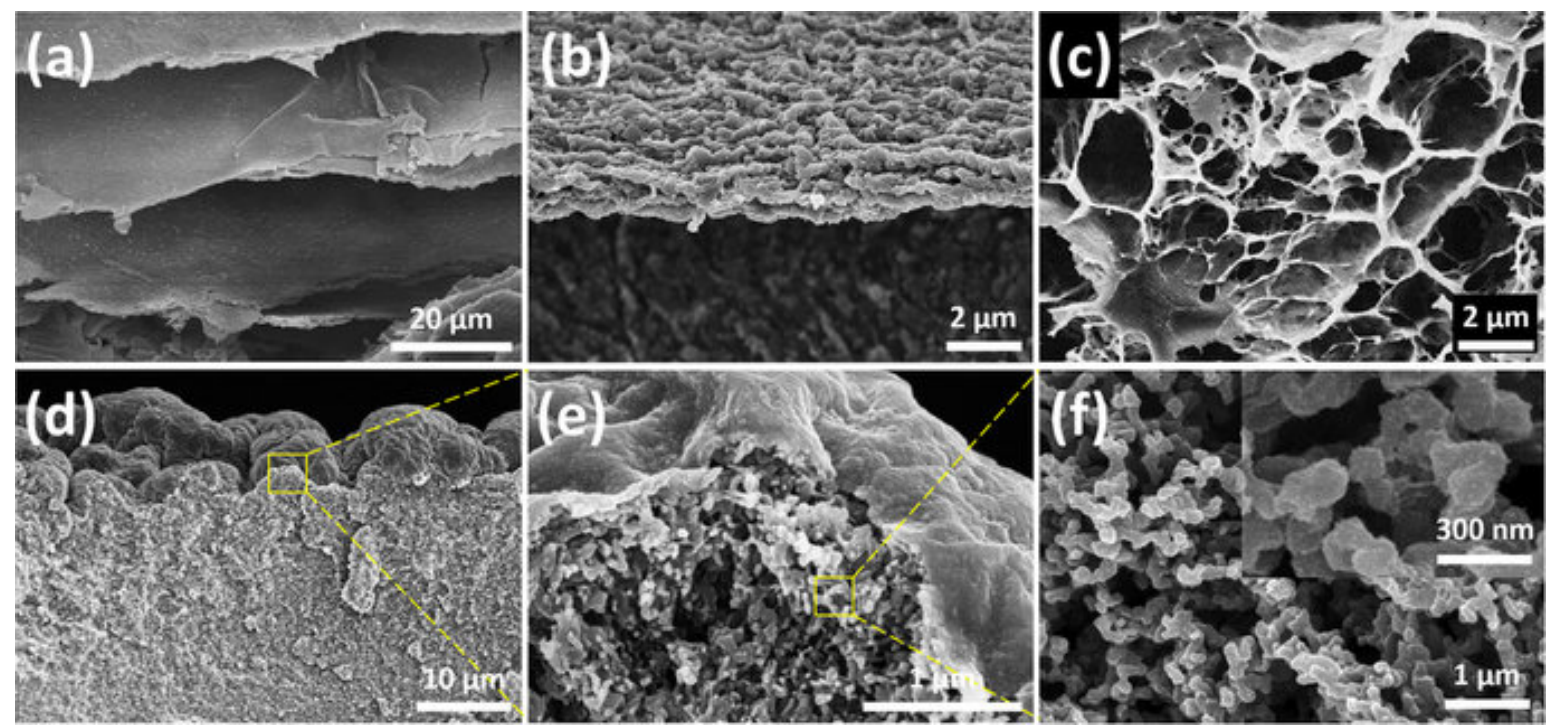

Figure 2. A cryo-fractured cross-section SEM images of (a,b) Ca-alginate, (c) alginate/AIP clay composite, and (d) P-MSC composite, (e) a magnified image of outer surface and inner structure of the P-MSC composite, and (f) close inspection of inner structure of the P-MSC composite[4]. 